\title{
Deficiency of biodegradable
} plastic-degrading enzyme production in a gene-deletion mutant of phyllosphere yeast, Pseudozyma antarctica defective in mannosylerythritol lipid biosynthesis

\author{
Azusa Saika ${ }^{1}$, Hideaki Koike 2 , Tohru Yarimizu ${ }^{3}$, Takashi Watanabe ${ }^{3,4}$, Hiroko Kitamoto $^{3}$ and Tomotake Morita ${ }^{1 *}$
}

\begin{abstract}
The basidiomycetous yeast Pseudozyma antarctica (currently designated Moesziomyces antarcticus) produces extracellular enzymes and glycolipids, including mannosylerythritol lipids (MELs), which are biosurfactants. Strain GB-4(0) of this species was previously isolated from rice husks and produces biodegradable plastic-degrading enzyme (Pseudozyma antarctica esterase; PaE). In this study, we generated a MEL biosynthesis-deficient strain ( $\triangle \mathrm{PaEMT1)}$ by deleting the gene PaEMT1, which is essential to MEL biosynthesis in strain GB-4(0). The resulting $\triangle P a E M T 1$ strain showed deficient PaE activity, and the corresponding signal was hardly detected in its culture supernatant through western blotting analysis using rabbit anti-PaE serum. On the other hand, the relative expression of the gene PaCLE1, encoding PaE, was identical between GB-4(0) and $\triangle \mathrm{PaEMT1}$ based on quantitative real-time PCR. When strain $\triangle \mathrm{PaEMT1}$ was grown in culture media supplemented with various surfactants, i.e., Tween20, BRIJ35 and TritonX-100, and MELs, PaE activity and secretion recovered. We also attempted to detect intracellular PaE using cell-free extract, but observed no signal in the soluble or insoluble fractions of $\triangle P a E M T 1$. This result suggested that the PaCLE1 gene was not translated to $\mathrm{PaE}$, or that expressed PaE was degraded immediately in $\triangle \mathrm{PaEMT1}$. Based on these results, MEL biosynthesis is an important contributor to PaE production.
\end{abstract}

Keywords: Pseudozyma antarctica, Esterase, Biodegradable plastic, Mannosylerythritol lipid, Glycosyltransferase, Gene deletion

\section{Introduction}

Pseudozyma antarctica (currently designated Moesziomyces antarcticus) has the ability to produce some materials, including enzymes and glycolipids (Boekhout and Fell 1998). In recent years, we have focused on an esterase (Pseudozyma antarctica esterase; PaE) that degrades biodegradable plastics (BP), including poly(butylene succinate) (PBS), poly(butylene succinate-co-adipate)

*Correspondence: morita-tomotake@aist.go.jp

${ }^{1}$ Research Institute for Sustainable Chemistry, National Institute of Advanced Industrial Science and Technology (AIST), Tsukuba Central 5-2, 1-1-1 Higashi, Tsukuba, Ibaraki 305-8565, Japan

Full list of author information is available at the end of the article
(PBSA), poly( $\varepsilon$-caprolactone) (PCL), and poly(lactide) (PLA) (Kitamoto et al. 2011; Shinozaki et al. 2013). P. antarctica strains isolated from rice husks secrete relatively high amounts of PaE into the culture supernatant, and $\mathrm{PaE}$ production is strongly enhanced when these strains are cultivated with xylose (Watanabe et al. 2014). PBSA, PBS and commercially available BP mulch films submerged in the culture supernatant of $P$. antarctica are rapidly degraded (Watanabe et al. 2014). To accelerate $\mathrm{PaE}$ utilization, we constructed a recombinant strain that is able to produce large amounts of $\mathrm{PaE}$ (13.4-fold higher amount than that of the wild-type strain) (Watanabe et al. 2016). Additionally, a targeted gene manipulation method has been developed recently (Yarimizu et al. 
2017) that allows more efficient modification of the $\mathrm{PaE}$ production of this strain.

Pseudozyma antarctica is well known to produce glycolipid-type biosurfactants known as mannosylerythritol lipids (MELs), which are composed of mannose, erythritol and fatty acids. MELs show excellent interfacial properties, as well as moisturizing activity toward cultured human skin cells; therefore, they have been used commercially in skincare products and cosmetics (Yamamoto et al. 2012). MEL production by $P$. antarctica was first reported in strain T-34, isolated from the exudate of a tree on Mt. Tsukuba in Japan (Kitamoto et al. 1990). The genomic sequence of $P$. antarctica T-34 has been reported, and five genes ( $\mathrm{Pa} E M T 1, \mathrm{~Pa} M A C 1, \mathrm{~Pa} M A C 2$, $\mathrm{Pa} M A T 1$ and $\mathrm{PaMMF1)}$ involved in MEL biosynthesis have been identified (Morita et al. 2013a).

Recently, we found that the BP film degradation activity of $\mathrm{PaE}$ was inhibited by MELs through in vitro analysis using surface plasmon resonance (Fukuoka et al. 2016). The hydrophobic lipid domain of MEL interacted with the BP film, and the hydrophilic sugar domains of MEL were regularly oriented on the MEL-coated film surface. The hydrophobic portion of $\mathrm{PaE}$ attaches to hydrophobic substrates such as BPs, as interaction between the enzyme and substrate is crucial for the degradation activity (Shinozaki et al. 2013). The negative effects of synthetic surfactants on production of esterases by yeasts have been studied in several previous papers. Namely, production of lipases, a subclass of esterases, by the yeasts Candida viswanathii and Yarrowia lipolytica was suppressed with the addition of either Tween80 or TritonX-100 (Almeida et al. 2013; Dominguez et al. 2003). Thus, we speculated that MELs biosynthesized by the host strain would also have negative effects on PaE production, and that deletion of MEL biosynthesis genes may eliminate these negative effects.

To remove the ability to MEL biosynthesis, we focused on the gene PaEMT1, encoding glycosyltransferase, because this gene is essential for MEL biosynthesis. A glycosyltransferase-deletion strain of the smut fungus Ustilago maydis and P. antarctica strain T-34 were completely defective in MEL biosynthesis (Hewald et al. 2005; Morita et al. 2010). In this study, we constructed a MEL biosynthesis-deficient strain through deletion of $\mathrm{PaEMT1}$ in GB-4(0). Unexpectedly, the PaEMT1-deletion strain was defective in the production of both MEL and PaE. The gene PaCLE1, encoding PaE, was expressed normally, regardless of MEL biosynthesis. The effect of MEL on $\mathrm{PaE}$ production was investigated in the $\mathrm{PaEMT1}$-deletion strain with and without exogenous surfactants, and the deficiency of enzyme production was recovered with the addition of MEL and other synthetic surfactants to the culture medium. Therefore, we speculated that either the PaCLE1 gene was not translated to $\mathrm{PaE}$ or expressed $\mathrm{PaE}$ was degraded immediately in strain $\triangle \mathrm{Pa} E M T 1$. This is the first report focused on their production between extracellular enzyme and glycolipid using a MEL biosynthesis deficient strain.

\section{Materials and methods}

\section{Strains and plasmid}

The strains and plasmids used in this study are listed in Table 1. Pseudozyma antarctica strain GB-4(0) (Kitamoto et al. 2011; Accession No. MAFF 306999) and PaEMT1deletion strain PaEMT1 $\triangle:$ NAT $(\triangle \mathrm{PaEMT1})$ of GB-4(0) were employed as the host strains for $\mathrm{PaE}$ production. The $\triangle \mathrm{PaEMT1}$ strain was generated as described below. PaEMT1 from the $P$. antarctica T-34 (Accession No. DF196785) expression plasmid pUXV1_neo-PaEMT1 was using for complementation of $\triangle \mathrm{Pa} E M T 1$ (Morita et al. 2013b).

\section{Construction of the MEL biosynthesis-deficient strain}

To obtain a strain that does not produce MEL, the gene $\mathrm{PaEMT1}$ was deleted through homologous recombination in $P$. antarctica GB-4(0). The two gene fragments upstream (1.1-kb) and downstream (1.0-kb) of PaEMT1 were amplified via PCR with the primer set (PaEMT1_ up_F1, R1 and PaEMT1_down_F1, R1) listed in Table 1, and with the genomic DNA of strain GB-4(0) used as a template. The natMX4 cassette with the nourseothricin acetyltransferase gene (nat1) controlled by Ashbya gossypii TEF promoter and terminator $(1.2-\mathrm{kb})$ was amplified through PCR with the primer set (NAT_F1, R1) listed in Table 1, using plasmid pAG25 (Goldstein and McCusker 1999) as a template. The gene fragment natMX4, flanked by the sequences of the PaEMT1 locus, was amplified by overlap PCR using three fragments that share 40 base-pair end-terminal homology as templates, yielding fragment PaEMT1::natMX4 (Additional file 1: Figure S1A), checked using gel electrophoresis, and purified with the MagExtractor-PCR \& Gel Clean-up kit (TOYOBO Co., Ltd., Osaka, Japan). Approximately $10 \mu \mathrm{g}$ of this DNA fragment was introduced into strain GB-4(0) using the lithium acetate method (Yarimizu et al. 2017). The transformed cells were grown on YPD medium agar plates containing $100 \mu \mathrm{g} / \mathrm{mL}$ nourseothricin (clonNAT; Jena Bioscience, Jena, Germany), and $\mathrm{PaEMT1}$ deletion was confirmed using colony PCR.

\section{PaE production}

Seed cultures of the strains were grown in $2 \mathrm{~mL} Y M$ medium ( $3 \mathrm{~g} / \mathrm{L}$ yeast extract, $3 \mathrm{~g} / \mathrm{L}$ malt extract, $5 \mathrm{~g} / \mathrm{L}$ peptone and $10 \mathrm{~g} / \mathrm{L}$ glucose) in test tubes at $30{ }^{\circ} \mathrm{C}$ for 3 days with shaking at 250 strokes/min (reciprocal). Then, $300 \mu \mathrm{L}$ of seed culture was inoculated into a $300-\mathrm{mL}$ 
Table 1 Strains, plasmids and primers used in this study

\begin{tabular}{|c|c|c|}
\hline Strains, plasmids, primers & Description & Reference/source \\
\hline \multicolumn{3}{|l|}{ Strains } \\
\hline P. antarctica GB-4(0) & Wild type strain isolated from rice husks and stocked in our laboratory & Kitamoto et al. (2011) \\
\hline P. antarctica $G B-4(0) \triangle P a E M T 1$ & PaEMT1 deleted strain & In this study \\
\hline \multicolumn{3}{|l|}{ Plasmids } \\
\hline pAG25 & $\begin{array}{l}\text { Dominant marker gene deletion cassette: pAgTEF1-natMX-tAgTEF1, clonNAT and } \\
\text { ampicillin resistance }\end{array}$ & $\begin{array}{l}\text { Goldstein and } \\
\text { McCusker (1999) }\end{array}$ \\
\hline pUXV1_neo & $\begin{array}{l}\text { Expression plasmid, glyceraldehyde-3P-dehydrogenase (gap) promoter, geneticin } \\
\text { (G418) and ampicillin resistance }\end{array}$ & Watanabe et al. (2016) \\
\hline pUXV1_neo-PaEMT1 & Inserted PaEMT1 from P. antarctica T-34 into pUVX1_neo & Morita et al. (2013b) \\
\hline \multicolumn{3}{|l|}{ Primers } \\
\hline PaEMT1_up_F1 & GAAGAGGCAATGGTGTTGCT & In this study \\
\hline PaEMT1_up_R1 & CGGCGGGGACAAGGCAAGCTATTTGCTTGAGCGATATGCTTTTTG & In this study \\
\hline NAT_F1 & AGCATATCGCTCAAGCAAATAGCTTGCCTTGTCCCCGCCGGGTCAC & In this study \\
\hline NAT_R1 & GTTTAATCAGGACAAGGCGTTCGACACTGGATGGCGGCGTTAGTATCG & In this study \\
\hline PaEMT1_down_F1 & ACGCCGCCATCCAGTGTCGAACGCCTTGTCCTGATTAAACTGCTG & In this study \\
\hline PaEMT1_down_R1 & CAATGTCATCCACAGCACTC & In this study \\
\hline PaEMT1_inner_F1 & CTGCATCGATTGATCCATTG & In this study \\
\hline PaEMT1_inner_R1 & GTCGAACCACTGCGACAGGA & In this study \\
\hline PaEMT1_up_F2 & ATTGCTGACCATCATGGTGC & In this study \\
\hline NATfragment_R1 & CACGTCAAGACTGTCAAGGA & In this study \\
\hline PaCLE1_qPCR_F & TTCCGGACCATGAACACCCG & In this study \\
\hline PaCLE1_qPCR_R & AGGAGGAAGCACGTGTTGGG & In this study \\
\hline Actin_qPCR_F & CAGTCGTCTGCGCTCGAGAA & In this study \\
\hline Actin_qPCR_R & CGGATGTCCAGGTCGCACTT & In this study \\
\hline
\end{tabular}

flask with $30 \mathrm{~mL}$ modified(m)-3 $\times \mathrm{FMM}$ (fungal minimum medium; $3 \mathrm{~g} / \mathrm{L}$ yeast extract, $2 \mathrm{~g} / \mathrm{L} \mathrm{NaNO}_{3}, 0.6 \mathrm{~g} / \mathrm{L}$ $\mathrm{KH}_{2} \mathrm{PO}_{4}$ and $\left.0.6 \mathrm{~g} / \mathrm{L} \mathrm{MgSO}_{4} \cdot 7 \mathrm{H}_{2} \mathrm{O}\right)$ containing $8(\mathrm{w} / \mathrm{v}) \%$ xylose that modified from previous report (Watanabe et al. 2014). The strains were cultivated at $30{ }^{\circ} \mathrm{C}$ for 4 days with 200 strokes/min shaking (rotary). After cultivation, the culture was centrifuged at $20,400 g$, and its supernatant was used for the PaE activity assay. To evaluate the functional complementation of $\triangle \mathrm{PaEMT1}, \mathrm{PaEMT1}$ was introduced using a plasmid that carries a bacterial neomycin resistance gene (neo), namely pUXV1_neoPaEMT1, via the lithium acetate method, and allowing selection on YM medium containing $500 \mu \mathrm{g} / \mathrm{mL}$ geneticin (G418). $200 \mu \mathrm{g} / \mathrm{mL}$ G418 was added to the culture medium to maintain the plasmid. Cell growth was determined by measuring the optical density of samples at $600 \mathrm{~nm}\left(\mathrm{OD}_{600}\right)$. All cultivations were performed in triplicate.

\section{PaE detection through SDS-PAGE and western blotting}

$\mathrm{PaE}$ production in the culture supernatant and cell-free extract were analyzed through sodium dodecyl sulfate polyacrylamide gel electrophoresis (SDS-PAGE) according to the method of Laemmli (1970), using a $14 \%$ polyacrylamide slab gel (culture supernatant) or Any kD Mini-Protean TGX gel (cell-free extract; Bio-Rad, Richmond, CA, USA). Proteins were visualized with Coomassie brilliant blue (CBB) staining using Quick-CBB (culture supernatant; FUJIFILM Wako Pure Chemical Corporation, Osaka, Japan) or SimplyBlue ${ }^{\mathrm{TM}}$ SafeStain (cell-free extract; Invitrogen, Carlsbad, CA, USA) kits. The proteins separated through SDS-PAGE were transferred to PVDF membranes using Trans-Blot SD Semi-Dry Transfer Cell (Bio-Rad) at $60 \mathrm{~mA}$ for $60 \mathrm{~min}$. Detection of PaE through western blot analysis was performed using rabbit anti-PaE serum $\left(1 / 3.5 \times 10^{4}\right)$ (Kitamoto et al. 2011) and HRP-linked donkey anti-rabbit polyclonal antibody (GE Healthcare, Little Chalfont, UK) as the primary and secondary antibodies, respectively. The PaE-antibody conjugate was detected with FluorChem and AlphaEase FC Software (Alpha Innotech, San Jose, CA, USA) using ECL western blotting detection reagent (GE Healthcare). Purified $\mathrm{PaE}$ was used as a control, as described previously (Suzuki et al. 2013).

\section{Preparation of cell-free extract}

Preparation of the cell-free extract is summarized in Additional file 1: Figure S2. To prepare cell-free extract, 
cells were harvested from $1 \mathrm{~mL}$ culture broth through centrifugation at $9100 \mathrm{~g}$ for $2 \mathrm{~min}$. The cells were resuspended in $0.5 \mathrm{~mL} 20 \mathrm{mM}$ Tris- $\mathrm{HCl}(\mathrm{pH} \mathrm{6.8)}$ containing $1 \mathrm{mM}$ PMSF, and then disrupted with two $5-\mathrm{mm}$ and twenty $2-\mathrm{mm}$ zirconia beads at $3200 \mathrm{rpm}$ for $5 \mathrm{~min}$ (three times) using the Beads Crusher $\mu \mathrm{T}-12$ (TAITEC, Saitama, Japan). After disruption, $0.5 \mathrm{~mL} 20 \mathrm{mM}$ Tris- $\mathrm{HCl}$ ( $\mathrm{pH} 6.8$ ) wad added, and $0.8 \mathrm{~mL}$ of sample was transferred to a $1.5-\mathrm{mL}$ tube. The cell debris was removed through centrifugation at $9100 \mathrm{~g}$ for $2 \mathrm{~min}$ and re-suspended in $0.8 \mathrm{~mL} 20 \mathrm{mM}$ Tris- $\mathrm{HCl}(\mathrm{pH} \mathrm{6.8)}$ as the insoluble fraction. TritonX-100 was added to the insoluble fraction (final concentration: $0.01 \%$ ) to treat cell debris, and the mixture was incubated at $30{ }^{\circ} \mathrm{C}$ for $1 \mathrm{~h}$ with $100 \mathrm{rpm}$ shaking (reciprocal). The treated cell debris was removed via centrifugation at $9100 \mathrm{~g}$ for $2 \mathrm{~min}$ and re-suspended in $20 \mathrm{mM}$ Tris $-\mathrm{HCl}(\mathrm{pH}$ 6.8) as the insoluble fraction after TritonX-100 treatment. Each sample was analyzed using SDS-PAGE and PaE was detected through western blot analysis.

\section{PaE activity}

Biodegradable plastic degradation activity was evaluated using emulsified PBSA (Bionolle EM-301; Showa Denko K. K., Tokyo, Japan), as described previously (Kitamoto et al. 2011). Briefly, a reaction mixture containing $20 \mathrm{mM}$ Tris- $\mathrm{HCl}$ (pH 6.8), 0.045 (w/v)\% emulsified PBSA and supernatant of the yeast culture or purified $\mathrm{PaE}$ was incubated at $30{ }^{\circ} \mathrm{C}$ for $15 \mathrm{~min}$ with 180 strokes/min shaking. After addition of the culture supernatant, the decrease in absorbance of the PBSA emulsion was measured at $660 \mathrm{~nm}\left(\mathrm{OD}_{660}\right)$. One unit (U) of PBSA degradation activity was defined as the activity that decrease $\mathrm{OD}_{660}$ by $1 \mathrm{in} 1 \mathrm{~mL}$ reaction solution in $1 \mathrm{~min}$. All analyses were performed in triplicate.

\section{Production and detection of MELs}

Cells were cultivated in MEL production medium $\left(1 \mathrm{~g} / \mathrm{L}\right.$ yeast extract, $3 \mathrm{~g} / \mathrm{L} \mathrm{NaNO}_{3}, 0.3 \mathrm{~g} / \mathrm{L} \mathrm{KH}_{2} \mathrm{PO}_{4}$ and $\left.0.3 \mathrm{~g} / \mathrm{L} \mathrm{MgSO}_{4} \cdot 7 \mathrm{H}_{2} \mathrm{O}\right)$ containing $2(\mathrm{w} / \mathrm{v}) \%$ soybean oil at $30{ }^{\circ} \mathrm{C}$ for 4 days. The MELs produced were detected using thin-layer chromatography (TLC), as described previously (Morita et al. 2007). The method can be summarized as follows: cell culture was mixed with an equal volume of ethyl acetate, and $10 \mu \mathrm{L}$ of this ethyl acetate extract was analyzed through TLC using chloroform: methanol: $12 \% \mathrm{NH}_{4} \mathrm{OH}=65: 15: 2(\mathrm{v} / \mathrm{v} / \mathrm{v})$ as an eluent. MELs were detected by spraying with $2 \%$ anthrone-sulfate reagent and heating at $90^{\circ} \mathrm{C}$ for $5 \mathrm{~min}$. A purified mixture of MEL-A, MEL-B and MEL-C was used as a reference.
The effects of surfactant addition on $\mathrm{PaE}$ production by strain $\triangle \mathrm{PaEMT1}$

$\triangle \mathrm{PaEMT1}$ was cultivated in $m-3 \times \mathrm{FMM}$ containing 8 (w/v)\% xylose supplemented with $0.01(\mathrm{w} / \mathrm{v}) \%$ of MELs, Tween20, BRIJ35, TritonX-100, SDS, sodium laurate $(\mathrm{C} 12 \mathrm{Na})$ or sodium stearate $(\mathrm{C} 18 \mathrm{Na})$. After 4 days of cultivation, the $\mathrm{PaE}$ activity in the culture supernatant was analyzed.

\section{Analysis of $\mathrm{PaCLE} 1$ expression levels through quantitative real-time PCR}

The gene expression levels of $\mathrm{PaCLE1}$, encoding $\mathrm{PaE}$, were analyzed through quantitative real-time PCR (qRTPCR). $P$. antarctica GB-4(0) and strain $\triangle \mathrm{PaEMT1}$ were cultivated in $m-3 \times$ FMM medium containing $8(\mathrm{w} / \mathrm{v}) \%$ xylose with or without $0.01(\mathrm{w} / \mathrm{v}) \%$ TritonX-100. After 3 days of cultivation, cells were harvested from $30 \mathrm{~mL}$ of culture by centrifugation at $800 \mathrm{~g}$ for $3 \mathrm{~min}$ and washed with distilled water twice. Total RNA was isolated from each sample with ISOGEN (Nippon Gene, Tokyo, Japan) according to the manufacturer's instructions, and the total concentration of purified RNA was determined using a Nanodrop fluorometer (Nano Drop Technologies, Wilmington, DE, USA). Then, mRNA was purified with the Oligotex-dT30 $<$ Super $>$ mRNA Purification Kit (Takara Bio, Shiga, Japan). cDNA was synthesized from a template of $50 \mathrm{ng}$ mRNA using the SuperScript VILO cDNA synthesis kit (Invitrogen) according to the manufacturer's instructions. Primers for qRT-PCR were designed using Primer 3 (http://bioinfo.ut.ee/prime r3-0.4.0/primer3/) (Table 1). The actin gene was used as a housekeeping gene. qRT-PCR was performed using Fast SYBR Green Master Mix (Applied Biosystems, Foster City, CA, USA) and visualized with LightCycler 2.0 with LightCycler software (ver. 5.0; Roche Diagnostics, Basel, Switzerland). Primer specificity was examined via melting curve analysis. Gene expression levels were quantified using the following equation: gene expression level $=2^{-[\mathrm{Ct}(\operatorname{PaCLE1})-\mathrm{Ct}(\mathrm{actin})]}$. The accession numbers of the actin gene and PaCLE1 are LC466625 and LC276896, respectively.

\section{Results}

\section{Deletion of PaEMT1 in strain GB-4(0)}

To obtain the MEL biosynthesis-deficient strain of GB-4(0), PaEMT1 was deleted and replaced with natMX4 through homologous recombination (Additional file 1: Figure S1A). The transformants generated on YPD plates with clonNAT were picked and analyzed using colony PCR to confirm PaEMT1 deletion (Additional file 1: Figure $\mathrm{S} 1 \mathrm{~B})$. The inner region $(1.0 \mathrm{~kb})$ of PaEMT1 was amplified via PCR with primers PaEMT1_inner_F1 
(Primer A) and PaEMT1_inner_R1 (Primer B) from the wild-type colonies, but not from the transformants. On the other hand, amplification of the gene fragment PaEMT1::natMX4 with primers PaEMT1_up_F2 (Primer C) and NATfragment_R1 (Primer D) was detected from the transformants. The transformants were defective in terms of MEL production when the cells were grown in MEL production medium, whereas the wild-type cells produced MELs (Fig. 1). These results indicate that PaEMT1 was replaced with natMX4, and that MEL biosynthesis was defective in the transformants, showing that $\mathrm{PaEMT1}$ is essential for MEL biosynthesis in GB-4(0).

\section{$\mathrm{PaE}$ activity of strain $\triangle \mathrm{PaEMT1}$}

To investigate the effect of PaEMT1 deletion on $\mathrm{PaE}$ production, strains GB-4(0) and PaEMT1-deletion strain $\mathrm{PaEMT} 1 \triangle:: \mathrm{NAT}(\triangle \mathrm{PaEMT1})$ were cultivated in $m-3 \times$ FMM- $8 \%$ xylose. Cell growth of $\triangle \mathrm{Pa} E M T 1$ was the same as that of the wild-type (Fig. 2a). Contrary to our expectation, $\mathrm{PaE}$ activity in the culture supernatant of $\triangle \mathrm{PaEMT1}(0.2 \pm 0.2 \mathrm{U} / \mathrm{mL}, 4$ days cultivation $)$ was clearly lower than that of the wild-type $(1.0 \pm 0.2 \mathrm{U} / \mathrm{mL}$, 4 days cultivation) (Fig. 2b). In SDS-PAGE and western blot analyses, the signal corresponding to PaE from $\triangle \mathrm{PaEMT1}$ was weaker than that from the wild-type (Fig. 2c). This result suggested that PaE expression and/or secretion decreased with defective MEL production. On the other hand, strain GB-4(0) secretes large amounts of $33-\mathrm{kDa}$ endo- $\beta$-xylanase in the presence of xylose (Watanabe et al. 2015). We observed similar signal intensity corresponding to xylanase in the culture supernatants of $\triangle \mathrm{PaEMT1}$ and wild-type cells (Fig. 2c). In addition, weak signals corresponding to other unknown proteins from $\triangle \mathrm{PaEMT1}$ were the same as those from the wild-type.

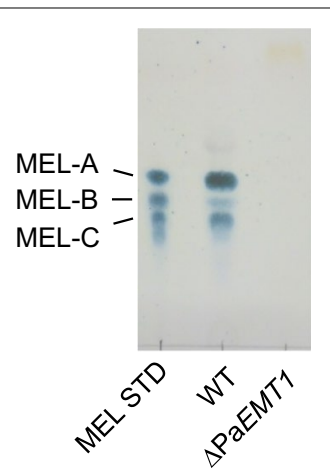

Fig. 1 PaEMT1 deletion through homologous recombination in P. antarctica GB-4(0). Thin-layer chromatography (TLC) analysis of mannosylerythritol lipid (MEL) production by wild-type and PaEMT1 $\triangle:$ :NAT ( $\triangle$ PaEMT1). MEL STD, MEL standard containing MEL-A, MEL-B and MEL-C
Based on these results, MEL biosynthesis is likely to contribute strongly to $\mathrm{PaE}$ production.

\section{Complementation of $\mathrm{PaEMT1}$ deletion}

To confirm the phenotypic changes due to loss of MEL production described above, PaEMT1 deletion was complemented through introduction of PaEMT1 with the plasmid pUXV1_neo-PaEMT1, and pUXV1_neo was used as a control. The recombinant strains $\triangle \mathrm{PaEMT1}$ harboring pUXV1_neo-PaEMT1 or pUXV1_neo were cultivated in MEL production medium containing 2 (w/v)\% soybean oil and $200 \mu \mathrm{g} / \mathrm{mL}$ G418 for 4 days at $30{ }^{\circ} \mathrm{C}$. In TLC analysis, MEL production was recovered in $\triangle \mathrm{PaEMT1}$ harboring pUXV1_neo-PaEMT1 (Fig. 3a). Cell growth of both strains was identical (Fig. 3b). PaE activity and secretion in the culture supernatant were recovered in $\triangle \mathrm{PaEMT1}$ harboring PUXV1_neo-PaEMT1 (Fig. 3b, c) in addition to MEL production. These results strongly support MEL biosynthesis being necessary for $\mathrm{PaE}$ secretion or production in strain GB-4(0).

\section{The effects of surfactant addition on PaE production in strain $\triangle \mathrm{PaEMT1}$}

We then investigated the effects of $0.01 \%$ MELs and various synthetic surfactants on $\mathrm{PaE}$ production in $\triangle \mathrm{PaEMT1}$. While SDS and $\mathrm{C} 12 \mathrm{Na}$ affected cell growth, the other additions (MELs, Tween20, BRIJ35, TritonX-100, C18Na) generally maintained normal growth of $\triangle \mathrm{PaEMT1}$ (Fig. 4a). The PaE activities in the cultures supplemented with Tween20, BRIJ35 and TritonX-100 were markedly elevated compared to the control (Fig. 4b). A signal corresponding to PaE was detected in all cultures supplemented with Tween20, BRIJ35 and TritonX-100 in SDS-PAGE and western blotting analyses (Fig. 4c). However, PaE activity and the PaE signal did not recover with $0.01 \%$ MEL addition, even though MEL is the native surfactant of strain GB-4(0).

The effect of varying the concentration of MELs was tested. A higher concentration of MELs did not affect cell growth (Fig. 5a). Recovery of PaE secretion was observed in the presence of 0.1 and $0.5 \%$ MELs based on PaE activity and PaE detection through western blotting (Fig. 5a, b). Based on this result, a higher concentration of MEL was required to recover $\mathrm{PaE}$ productivity compared to other surfactants.

\section{Relative expression level of PaCLE1 in $\triangle \mathrm{PaEMT1}$ strain}

Although the protein secretion/degradation pathway is unknown in $P$. antarctica, we analyzed the expression of $\mathrm{PaCLE1}$, encoding PaE, through qRT-PCR to determine whether $\mathrm{PaE}$ production deficiency depends on transcriptional regulation or an unknown protein degradation pathway. The expression level of the gene was the 

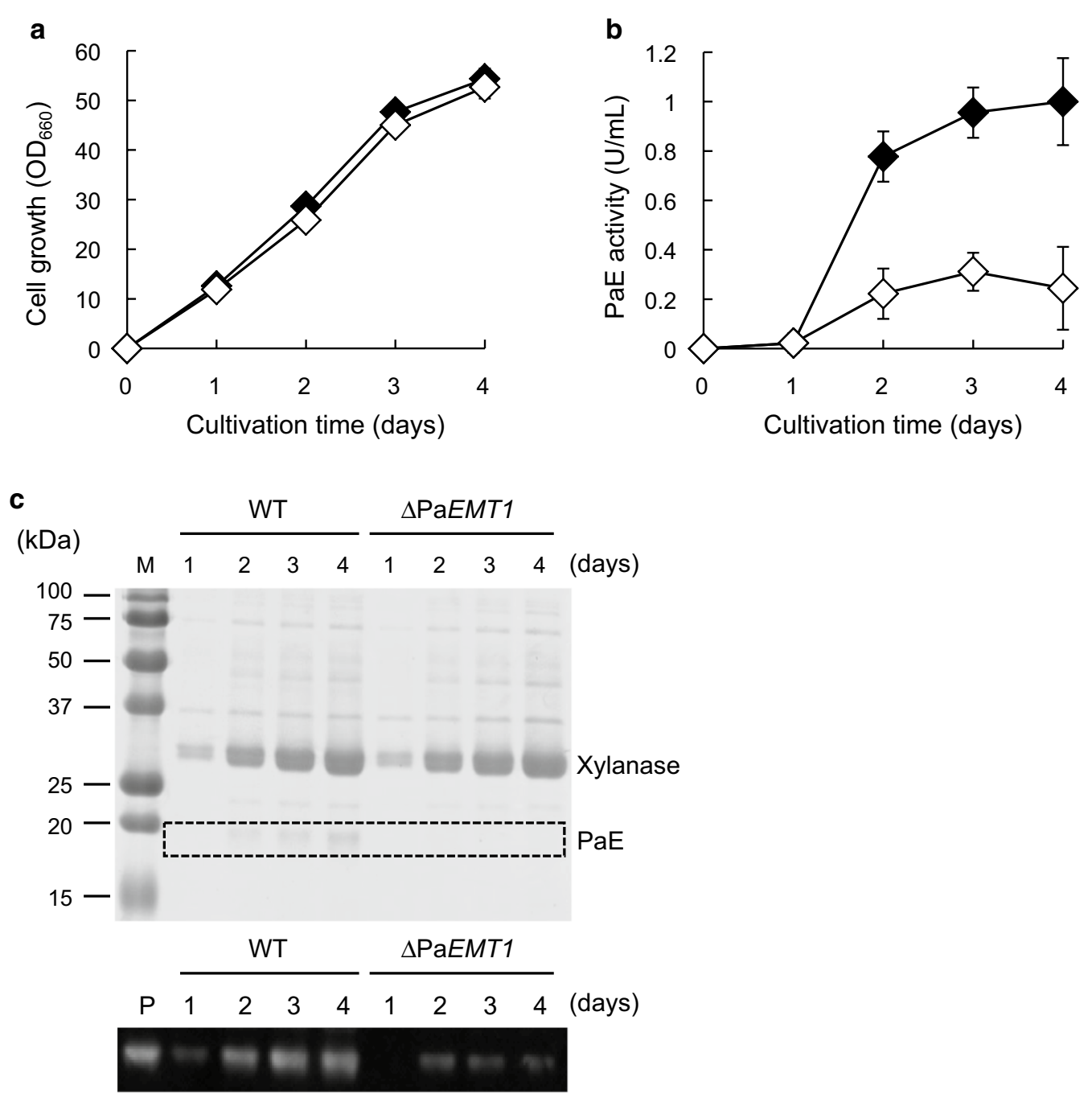

Fig. 2 Time course of PaE production by strain $\triangle \mathrm{PaEMT1}$ in $m-3 \times \mathrm{FMM}$ (fungal minimum medium) supplemented with $8 \%$ xylose. a Cell growth of wild-type (closed diamonds) and $\triangle \mathrm{PaEMT1}$ (open diamonds). b Plastic-degrading enzyme (PaE) activity of wild-type (closed diamonds) and $\triangle P a E M T 1$ (open diamonds). c sodium dodecyl sulfate polyacrylamide gel electrophoresis (SDS-PAGE) and PaE detection through western blot analysis of culture supernatants of wild-type and $\triangle P a E M T 1$. M, marker; P, purified PaE loaded as a standard. The amount of each culture supernatant loaded in the gel was $10 \mu \mathrm{L}$ for Coomassie brilliant blue (CBB) staining and western blotting. The results of the cell growth and PaE activity assays are shown as the average of three different experiments. Error bars show standard deviations

same in $\triangle \mathrm{PaEMT1}(2.26 \pm 0.22)$, wild-type $(2.12 \pm 0.25)$ and $\triangle \mathrm{Pa} E M T 1$ grown in the medium supplemented with $0.01 \%$ TritonX-100 (2.10 \pm 0.51$)$. This result indicates that $\mathrm{PaCLE1}$ is transcribed normally in $\triangle \mathrm{PaEMT1}$, but that the gene product secretion system was defective.

\section{Detection of $\mathrm{PaE}$ in cell-free extract}

As mentioned above, $\mathrm{PaE}$ was not secreted into the culture supernatant of $\triangle \mathrm{PaEMT1}$, although the $\mathrm{PaCLE1}$ gene was expressed at the same level as in the wild-type. From these results, we speculated that $\mathrm{PaE}$ secretion failed due to the loss of MEL production.
To test this hypothesis, cell-free extract was prepared and analysis of intracellular PaE was carried out using SDS-PAGE and western blotting. The detailed preparation method for cell-free extract is summarized in Additional file 1: Figure S2. According to Fig. 6, PaE was detected only in the culture supernatant of strain GB-4(0) (Fig. 6b, Lane 1), and showed no signals in the soluble or insoluble fractions of either strain GB-4(0) (Fig. 6b, Lane 2 and 3) or $\triangle \mathrm{PaEMT1}$ (Fig. 6b, Lane 7 and 8 ). Additionally, precipitate of the insoluble fraction was washed using $0.01 \%$ TritonX-100 for $1 \mathrm{~h}$ at $30{ }^{\circ} \mathrm{C}$ (Additional file 1: Figure S2). Because $\mathrm{PaE}$ 

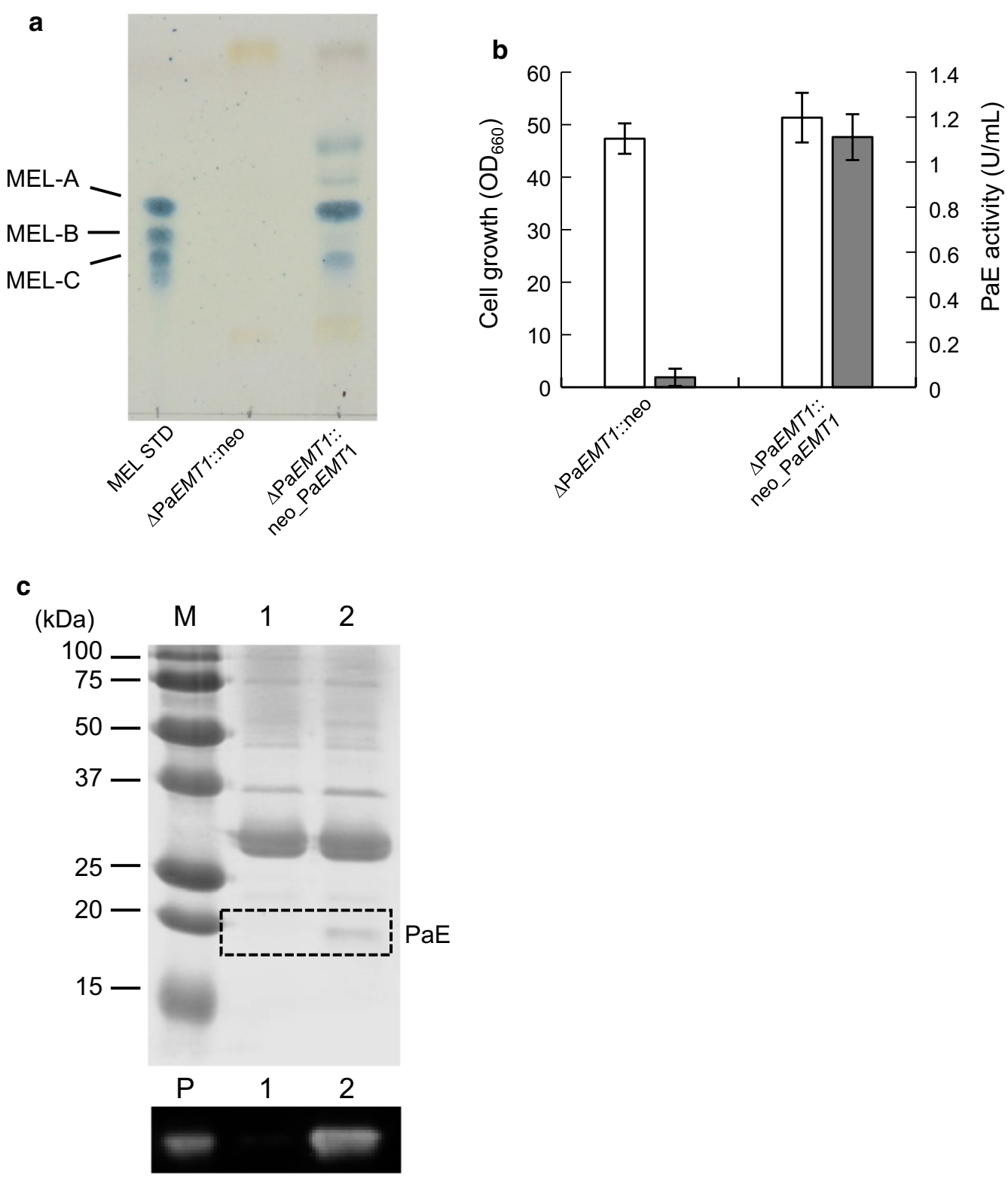

\section{1: $\triangle \mathrm{PaEMT1::neo,} \mathrm{2:} \triangle \mathrm{PaEMT1::PaEMT1}$}

Fig. 3 PaE production by strain $\triangle P a E M T 1$ harboring PaEMT1 in 3xFMM medium supplemented with 8\% xylose. a TLC analysis of MEL production by

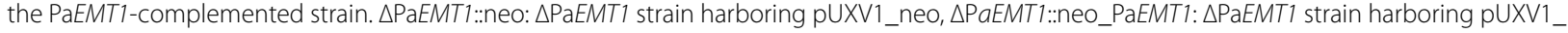
neo-PaEMT1. b Cell growth (white) and PaE activity (gray). c SDS-PAGE and PaE detection through western blot analysis of culture supernatant. M,

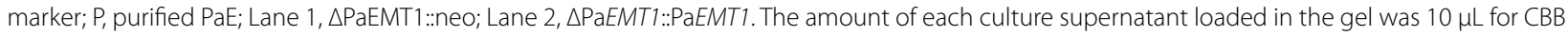
staining and western blotting. The results of the cell growth and PaE activity assays are shown as the average of three different experiments. Error bars show standard deviations

productivity recovered with the addition of $0.01 \%$ TritonX-100 (Fig. 4), we expected that PaE would be eluted after $0.01 \%$ TritonX-100 treatment if $\mathrm{PaE}$ was insolubilized. However, no signal was present in the fraction obtained after TritonX-100 treatment (Fig. 6b,
Lane 9 and 10). These results suggest that the gene $\mathrm{PaCLE1}$ was not translated to $\mathrm{PaE}$ or that expressed $\mathrm{PaE}$ was degraded immediately in $\triangle \mathrm{Pa} E M T 1$. 

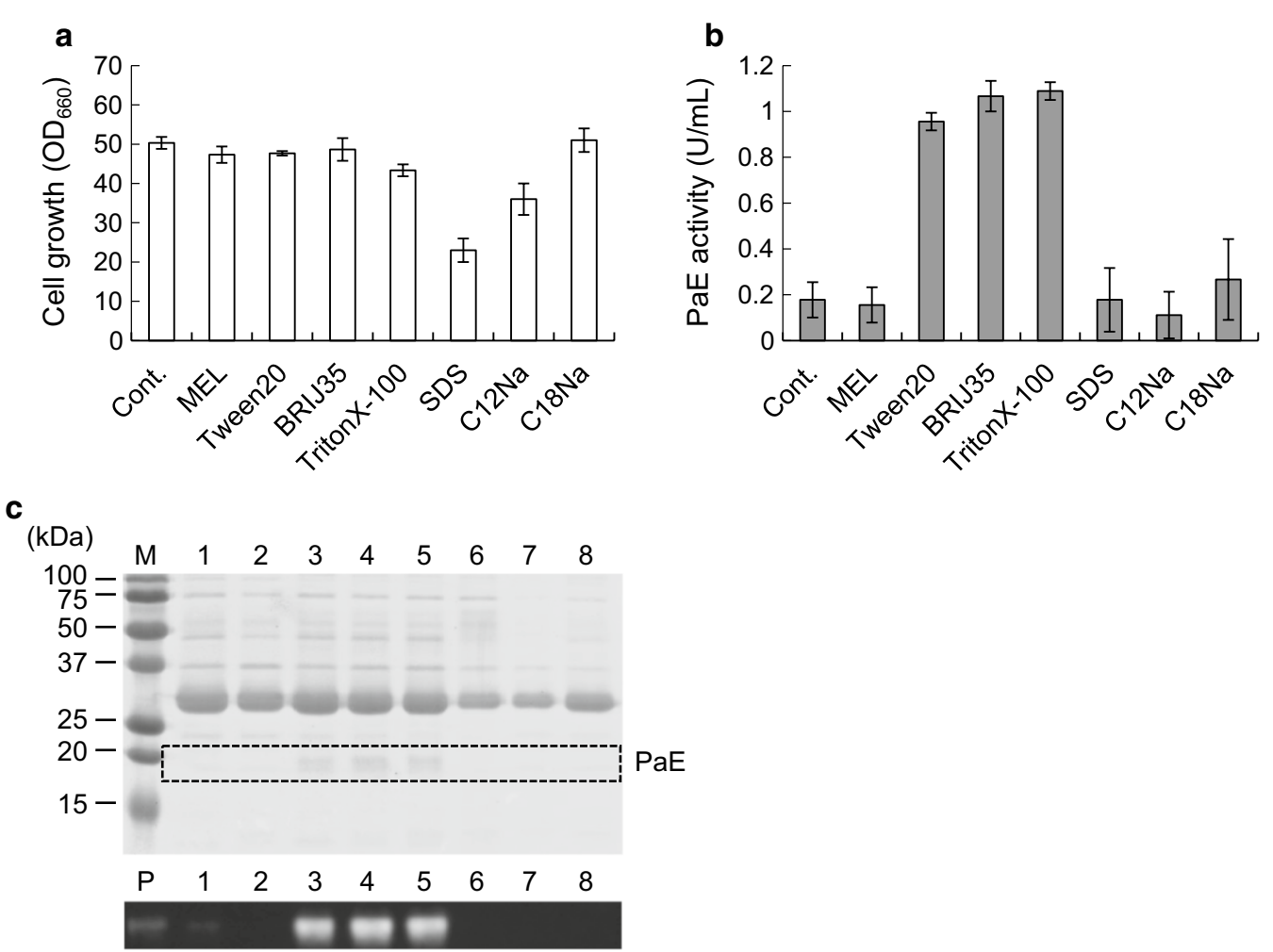

1: Cont., 2: MEL, 3: Tween20, 4: BRIJ35

5: TritonX-100, 6: SDS, 7: C12Na, 8: C18Na

Fig. 4 PaE production by strain $\triangle P a E M T 1$ in $m-3 \times F M M$ medium supplemented with $8 \%$ xylose and $0.01 \%$ surfactants. a Cell growth. b PaE activity. c SDS-PAGE and PaE western blot analysis of culture supernatant. M, marker; P, purified PaE; Lane 1, control; Lane 2, MEL; Lane 3, Tween20; Lane 4, BRIJ35; Lane 5, TritonX-100; Lane 6, SDS; Lane 7, sodium dodecanoate (C12Na); Lane 8, sodium octadecenoate (C18Na). The amount of each culture supernatant loaded in the gel was $10 \mu \mathrm{L}$ for CBB staining and western blotting. The results of the cell growth and PaE activity assays are shown as the average of three different experiments. Error bars show standard deviations

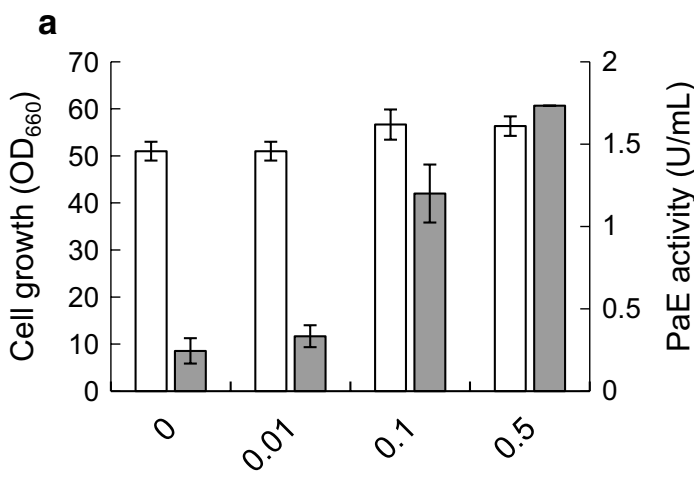

MEL concentration (\%) b

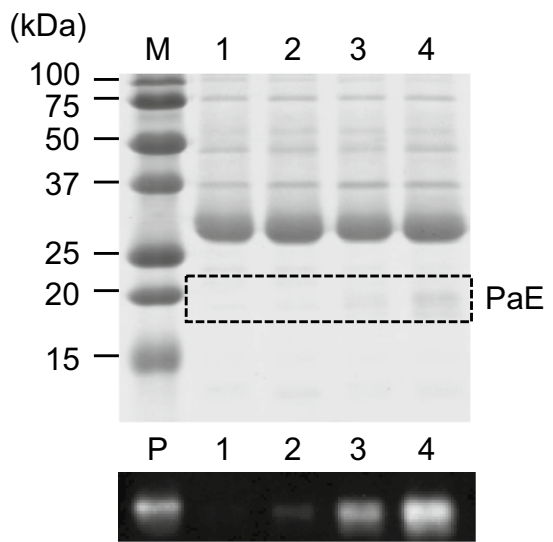

1: $0 \%, 2: 0.01 \%, 3: 0.1 \%, 4: 0.5 \%$

Fig. 5 PaE production by strain $\triangle P A E M T 1$ in $m-3 \times F M M$ medium supplemented with $8 \%$ xylose and various concentrations of MEL. a Cell growth (white) and PaE activity (gray). b SDS-PAGE and western blot analysis of culture supernatant. M, marker; P, purified PaE. The amount of each culture supernatant loaded in the gel was $10 \mu \mathrm{L}$ for CBB staining and western blotting. The results of the cell growth and PaE activity assays are shown as the average of three different experiments. Error bars show standard deviations 


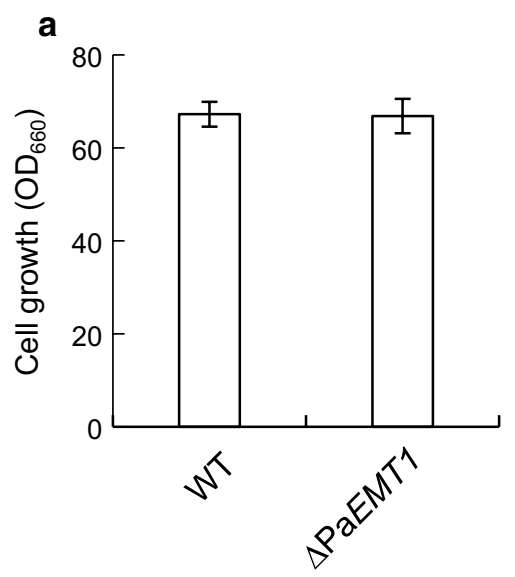

b

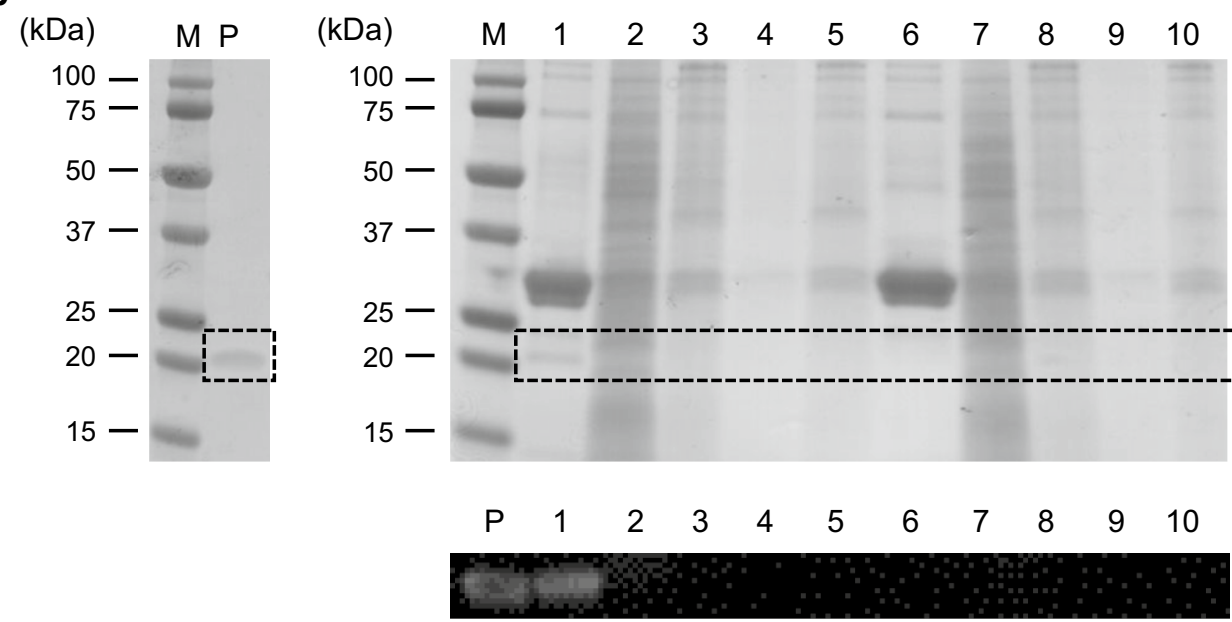

Supernatant of 1: WT, 6: $\triangle \mathrm{PaEMT1}$, Soluble fraction of 2: WT, $7: \triangle \mathrm{PaEMT1}$, Insoluble fraction of 3: WT, 8: $\triangle \mathrm{PaEMT1}$

Soluble fraction of after $0.01 \%$ TritonX-100 treatment of 4: WT, 9: $\triangle \mathrm{PaEMT1}$ Insoluble fraction of after $0.01 \%$ TritonX-100 treatment of 5: WT, 10: $\triangle \mathrm{PaEMT1}$

Fig. 6 SDS-PAGE and PaE western blot analysis of culture supernatant and cell-free extract of GB-4(0) and strain $\triangle P a E M T 1$. a Cell growth. b SDS-PAGE and PaE detection through western blot analysis. M, marker; P, purified PaE; Lane 1, supernatant of wild-type; Lane 2, soluble fraction of wild-type; Lane 3, insoluble fraction of wild-type; Lane 4, soluble fraction after 0.01\% TritonX-100 treatment of wild-type; Lane 5, insoluble fraction after $0.01 \%$ TritonX-100 treatment of wild-type; Lane 6, supernatant of $\triangle P a E M T 1$; Lane 7, soluble fraction of $\triangle \mathrm{PaEMT1}$; Lane 8, insoluble fraction of $\triangle P a E M T 1$; Lane 9, soluble fraction after $0.01 \%$ TritonX-100 treatment of $\triangle P a E M T 1$; Lane 10, insoluble fraction after $0.01 \%$ TritonX-100 treatment of $\triangle \mathrm{PaEMT1}$. The amount of each sample loaded in the gel was $20 \mu \mathrm{L}$ for CBB staining and western blotting. Error bars show standard deviations

\section{Discussion}

In this study, we found that the biosynthesis of extracellular glycolipids known as MELs contributes to production of an extracellular esterase, PaE, in P. antarctica GB-4(0). Furthermore, we demonstrated that addition of various surfactants including MEL complement this deficiency; thus, MEL biosynthesis is required for $\mathrm{PaE}$ production in strain GB-4(0).
A gene cluster consisting of five genes responsible for MEL biosynthesis has been reported in $U$. maydis UM521 and P. antarctica T-34, $P$. antarctica JCM10317 ${ }^{\mathrm{T}}$, P. aphidis DSM70725 and P. tsukubaensis NBRC1940 (Hewald et al. 2006; Lorenz et al. 2014; Morita et al. 2013a; Saika et al. 2014, 2016). Of these genes, a gene encoding glycosyltransferase (corresponding to Emt1 of $U$. maydis and PaEmt1p of $P$. antarctica) is essential for 
MEL production in $U$. maydis UM521 and $P$. antarctica T-34, as reported previously (Hewald et al. 2005; Morita et al. 2010). PaEMT1 from strain GB-4(0) shares high amino acid sequence identity with $U$. maydis UM521, $P$. antarctica T-34 and P. antarctica JCM10317; 75, 100 and 95\%, respectively. The PaEMT1-deletion strain exhibits MEL biosynthesis deficiency, and the phenotype was complemented by transformation with PaEMT1. Thus, $\mathrm{PaEMT1}$ is essential for MEL production in GB-4(0), in accordance with results from strain T-34 (Morita et al. 2010).

$\mathrm{PaE}$ activity recovered with the addition of synthesized 0.01\% Tween20, BRIJ35, or TritonX-100, while the other surfactants, including $0.01 \% \mathrm{MEL}$, did not lead to such activity (Fig. 4). The critical micelle concentration (CMC) of each surfactant was as follows: MEL: $0.0027 \mathrm{mM}$ $\left(1.8 \times 10^{-4} \%\right)$, Tween20: $0.06 \mathrm{mM}(0.0074 \%)$, BRIJ35: $0.09 \mathrm{mM}(0.011 \%)$, TritonX-100: $0.24 \mathrm{mM}(0.015 \%)$, SDS: $8 \mathrm{mM}(0.23 \%), \mathrm{C} 12 \mathrm{Na}: 27.5 \mathrm{mM}(0.61 \%)$, and C18Na: $0.5 \mathrm{mM}(0.015 \%)$. A concentration of $0.01 \%$ is lower than the CMC for BRIJ35, TrironX-100, SDS, C12Na and $\mathrm{C} 18 \mathrm{Na}$; therefore, CMC is likely unrelated to the recovery of $\mathrm{PaE}$ production. In addition, we estimated the effect of the initial content of TritonX-100 on PaE production with addition of $0,0.2,0.5,1$, and $5 \mathrm{CMC}$. As shown in Additional file 1: Figure S3, recovery of $\mathrm{PaE}$ production was observed at $0.2-5 \mathrm{CMC}$, supporting the hypothesis described above. SDS, $\mathrm{C} 12 \mathrm{Na}$ and $\mathrm{C} 18 \mathrm{Na}$, which did not have an effect on recovery of $\mathrm{PaE}$, are categorized as anionic surfactants, while MEL, Tween20, BRIJ35 and TritonX-100 are categorized as non-ionic surfactant. From this result, the type of surfactant might affect the ability to recover PaE production. According to Fig. 5, a higher concentration of MEL was needed to recover PaE production. P. antarctica is known to produce large amounts of MEL (over $14 \%$ in culture medium) (Kitamoto et al. 2001), which could be related to the requirement for a high concentration of MEL, but further investigation is needed to understand the mechanism of $\mathrm{PaE}$ secretion.

Fukuoka et al. (2016) showed that PaE activity decreased with the addition of MELs at final concentrations of 5 and $50 \mathrm{mg} / \mathrm{L}$ ( 0.0005 and $0.005 \%$, respectively). While the MEL concentrations shown in Fig. 5 were 1000 times higher than those in previous reports, $\mathrm{PaE}$ activities were not inhibited. The culture supernatant used for the PaE activity assay shown in Fig. 5 was analyzed through TLC (Additional file 1: Figure S4). According to the results, the added MEL was not detected in the supernatant of every culture (Additional file 1: Figure S4A). On the other hand, added MEL was detected in the precipitate that included cells, but the amount of MEL was lower than the MEL standard (Additional file 1: Figure S4B). Based on these results, addition of MEL did not inhibit PaE activity because the MEL was precipitated during centrifugation, and some of the added MEL may have degraded during cultivation.

As the PaCLE1 expression level of strain $\triangle \mathrm{PaEMT1}$ was the same as that of the parent strain, recovery of deficient $\mathrm{PaE}$ production with surfactants may be linked to post-translational events, such as protein trafficking and degradation pathways. Because these amphiphilic compounds will interact with the cell membrane, various intracellular pathways related to cell integrity, e.g., biosynthesis of components of the cell membrane and cell wall and the protein kinase $C$ pathway, respond to treatment with detergents. Moreover, the absence of MELs may induce deficiency of protein trafficking in transport vesicles, because the composition of intracellular membranes affects their function (Phillips et al. 2009; Alexander et al. 2011; Iwamoto and Oiki 2013). After incorporation into the intracellular membrane via endocytosis, the amphiphilic compounds may complement the absence of MELs in the intracellular membrane, although the function of MELs in protein sorting is unknown. Further study of the molecular cell biology of $P$. antarctica, including protein trafficking, degradation, secretion, and the localization and transportation pathways of MELs, will clarify the mechanisms of $\mathrm{PaE}$ production.

\section{Additional file}

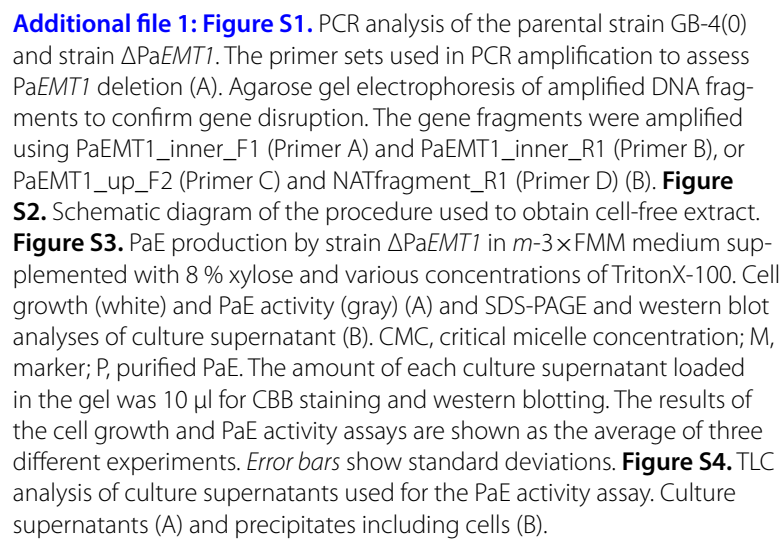
and strain $\triangle P a E M T 1$. The primer sets used in PCR amplification to assess PaEMT1 deletion (A). Agarose gel electrophoresis of amplified DNA fragments to confirm gene disruption. The gene fragments were amplified using PaEMT1_inner_F1 (Primer A) and PaEMT1_inner_R1 (Primer B), or PaEMT1_up_F2 (Primer C) and NATfragment_R1 (Primer D) (B). Figure S2. Schematic diagram of the procedure used to obtain cell-free extract. Figure S3. PaE production by strain $\triangle \mathrm{PaEMT1}$ in $m-3 \times \mathrm{FMM}$ medium supplemented with $8 \%$ xylose and various concentrations of TritonX-100. Cell growth (white) and PaE activity (gray) (A) and SDS-PAGE and western blot analyses of culture supernatant (B). CMC, critical micelle concentration; $M$, marker; $\mathrm{P}$, purified $\mathrm{PaE}$. The amount of each culture supernatant loaded in the gel was $10 \mu \mathrm{l}$ for CBB staining and western blotting. The results of the cell growth and PaE activity assays are shown as the average of three different experiments. Error bars show standard deviations. Figure S4. TLC analysis of culture supernatants used for the PaE activity assay. Culture supernatants (A) and precipitates including cells (B).

\section{Acknowledgements}

Not applicable.

\section{Authors' contributions}

AS designed the study, carried out most of the biological studies and drafted the manuscript. HK (H. Koike) carried out gene analysis and evaluated and discussed the results. TY and TW helped in the generation of $\triangle P a E M T 1$ and evaluated and discussed the results. HK (H. Kitamoto) and TM conceived and designed the study, and drafted the manuscript. All authors read and approved the final manuscript. 


\section{Funding}

This research was financially supported by a grant from the Science and Technology Research Promotion Program (25017A, 25017AB) for the agriculture, forestry, fisheries, and food industries.

\section{Availability of data and materials}

All data generated during this study are included in this article and its additional files.

\section{Ethics approval and consent to participate}

This article does not contain any studies with human participants or animals performed by any of the authors.

\section{Consent for publication}

Not applicable.

\section{Competing interests}

The authors declare that they have no competing interests.

\begin{abstract}
Author details
${ }^{1}$ Research Institute for Sustainable Chemistry, National Institute of Advanced Industrial Science and Technology (AIST), Tsukuba Central 5-2, 1-1-1 Higashi, Tsukuba, Ibaraki 305-8565, Japan. ${ }^{2}$ Bioproduction Research Institute, National Institute of Advanced Industrial Science and Technology (AIST), Tsukuba Central 6-9, 1-1-1 Higashi, Tsukuba, Ibaraki 305-8566, Japan. ${ }^{3}$ Institute for Agro-Environmental Sciences, National Agricultural Food Research Organization (NARO), 3-1-3 Kannondai, Tsukuba, Ibaraki 305-8604, Japan. ${ }^{4}$ Present Address: Gunma Industrial Technology Center, 884-1 Kamesato, Maebashi, Gunma 379-2147, Japan
\end{abstract}

Received: 19 June 2019 Accepted: 26 June 2019

Published online: 06 July 2019

\section{References}

Alexander RT, Jaumouillé V, Yeung T, Furuya W, Peltekova I, Boucher A, Zasloff M, Orlowski J, Grinstein S (2011) Membrane surface charge dictates the structure and function of the epithelial $\mathrm{Na}+/ \mathrm{H}+$ exchanger. EMBO J 30:679-691

Almeida AF, Tauk-Tornisielo SM, Carmona EC (2013) Acid lipase from Candida viswanathii: production, biochemical properties, and potential application. Biomed Res Int 2013, 435818

Boekhout T, Fell JW (1998) Pseudozyma Bandoni emend. Boekhout and a comparison with the yeast state of Ustilago maydis (de Candolle) Corda. In: Kurtzman CP, Fell JW (eds) The yeasts: a taxonomic study, 4th edn. Elsevier Science, Amsterdam, pp 790-797

Dominguez A, Deive FJ, Sanromán Longo MA (2003) Effect of lipids and surfactants on extracellular lipase production by Yarrowia lipolytica. J Chem Technol Biotechnol 78:1166-1170

Fukuoka T, Shinozaki Y, Tsuchiya W, Suzuki K, Watanabe T, Yamazaki T, Kitamoto D, Kitamoto H (2016) Control of enzymatic degradation of biodegradable polymers by treatment with biosurfactants, mannosylerythritol lipids, derived from Pseudozyma spp. yeast strains. Appl Microbiol Biotechnol 100:1733-1741

Goldstein AL, McCUSKER JH (1999) Three new dominant drug resistance cassettes for gene disruption in Saccharomyces cerevisiae. Yeast 15:1541-1553

Hewald S, Josephs K, Bölker M (2005) Genetic analysis of biosurfactant production in Ustilago maydis. Appl Environ Microbiol 71:3033-3040

Hewald S, Linne U, Scherer M, Marahiel MA, Kämper J, Bölker M (2006) Identification of a gene cluster of biosynthesis of mannosylerythritol lipids in the basidiomycetous fungus Ustilago maydis. Appl Environ Microbiol 72:5469-5477

Iwamoto M, Oiki S (2013) Amphipathic antenna of an inward rectifier K+ channel responds to changes in the inner membrane leaflet. PNAS 110:749-754

Kitamoto D, Akiba S, Hioki C, Tabuchi T (1990) Extracellular accumulation of mannosylerythritol lipids by a strain of Candida antarctica. Agric Biol Chem 54:31-36

Kitamoto D, Ikegami T, Suzuki GT, Sasaki A, Takeyama Y, Idemoto Y, Koura N, Yanagishita H (2001) Microbial conversion of $n$-alkanes into glycolipid biosurfactants, mannosylerythritol lipids, by Pseudozyma (Candida antarctica). Biotechnol Lett 23:1709-1714
Kitamoto HK, Shinozaki Y, Cao XH, Morita T, Konishi M, Tago K, Kajiwara H, Koitabashi M, Yoshida S, Watanabe T, Sameshima-Yamashita Y, Nakajima-Kambe T, Tsushima S (2011) Phyllosphere yeasts rapidly break down biodegradable plastics. AMB Express 1:44

Laemmli UK (1970) Cleavage of structural proteins during the assembly of the head of bacteriophage T4. Nature 277:680-685

Lorenz S, Guenther M, Grumaz C, Rupp S, Zibek S, Sohn K (2014) Genome sequence of the basidiomycetous fungus Pseudozyma aphidis DSM70725, an efficient producer of biosurfactant mannosylerythritol lipids. Genome Announc 2:e00053-14

Morita T, Konishi M, Fukuoka T, Imura T, Kitamoto HK, Kitamoto D (2007) Characterization of the genus Pseudozyma by the formation of glycolipid biosurfactants, mannosylerythritol lipids. FEMS Yeast Res 7:286-292

Morita T, Ito E, Kitamoto HK, Takegawa K, Fukuoka T, Imura T, Kitamoto D (2010) Identification of the gene PaEMT1 for biosynthesis of mannosylerythritol lipids in the basidiomycetous yeast Pseudozyma antarctica. Yeast 27:905-917

Morita T, Koike H, Koyama Y, Hagiwara H, Ito E, Fukuoka T, Imura T, Machida M, Kitamoto D (2013a) Genome sequence of the basidiomycetous yeast Pseudozyma antarctica T-34, a producer of the glycolipid biosurfactants mannosylerythritol lipids. Genome Announc 1:e00064-13

Morita T, Fukuoka T, Imura T, Kitamoto D (2013b) Production of mannosylerythtirol lipids and their application in cosmetics. Appl Microbiol Biotechnol 97:4691-4700

Phillips R, Ursell T, Wiggins P, Sens P (2009) Emerging roles for lipids in shaping membrane-protein function. Nature 459:379-385

Saika A, Koike H, Hori T, Fukuoka T, Sato S, Habe H, Kitamoto D, Morita T (2014) Draft genome sequence of the yeast Pseudozyma antarctica type strain JCM10317, a producer of the glycolipid biosurfactants, mannosylerythritol lipids. Genome Announc 2:e00878-14

Saika A, Koike H, Fukuoka T, Yamamoto S, Kishimoto T, Morita T (2016) A gene cluster for biosynthesis of mannosylerythritol lipids consisted of 4-O- $\beta$-Dmannopyranosyl-(2R,3S)-erythritol as the sugar moiety in a basidiomycetous yeast Pseudozyma tsukubaensis. PLoS ONE 11(6):e0157858

Shinozaki Y, Motira T, Cao XH, Yoshida S, Koitabashi M, Watanabe T, Suzuki K, Sameshia-Yamashita Y, Nakajima-Kambe T, Fujii T, Kitamoto HK (2013) Biodegradable plastic-degrading enzyme from Pseudozyma antarctica: cloning, sequencing, and characterization. Appl Microbiol Biotechnol 97:2951-2959

Suzuki K, Sakamoto H, Shinozaki Y, Tabata J, Watanabe T, Mochizuki A, Koitabashi M, Fujii T, Tsushima S, Kitamoto HK (2013) Affinity purification and characterization of a biodegradable plastic-degrading enzyme from a yeast isolated from the larval midgut of a stag beetle, Aegus laevicollis. Appl Microbiol Biotechnol 97:7679-7688

Watanabe T, Shinozaki Y, Yoshida S, Koitabashi M, Sameshia-Yamashita Y, Fujii T, Fukuoka T, Kitamoto HK (2014) Xylose induces the phyllosphere yeast Pseudozyma antarctica to produce a cutinase-like enzyme which efficiently degrades biodegradable plastics. J Biosci Bioeng 117:325-329

Watanabe T, Suzuki K, Sato I, Morita T, Koike G, Shinozaki Y, Ueda H, Koitabashi M, Kitamoto HK (2015) Simultaneous bioethanol distillery wastewater treatment and xylanase production by the phyllosphere yeast Pseudozyma antarctica GB-4(0). AMB Exprerss 5:36

Watanabe T, Morita T, Koike H, Yarimizu T, Shinozaki Y, Sameshia-Yamashita Y, Yoshida S, Koitabashi M, Kitamoto K (2016) High-level recombinant protein production by the basidiomycetous yeast Pseudozyma antarctica under a xylose-inducible xylanase promoter. Appl Microbiol Biotechnol 100:3207-3217

Yamamoto S, Morita T, Fukuoka T, Imura T, Yanagidani S, Sogabe A, Kitamoto D, Kitagawa M (2012) The moisturizing effects of glycolipid biosurfactants, mannosylerythritol lipids, on human skin. J Oleo Sci 61:407-412

Yarimizu T, Shimoi H, Sameshima-Yamashita Y, Morita T, Koike H, Watanabe T, Kitamoto H (2017) Targeted gene replacement at the URA3 locus of the basidiomycetous yeast Pseudozyma antarctica and its transformation using lithium acetate treatment. Yeast 34:483-494

\section{Publisher's Note}

Springer Nature remains neutral with regard to jurisdictional claims in published maps and institutional affiliations. 